\title{
Extremal orders of some functions connected to regular integers modulo $n$
}

\section{Brădut Apostol}

\begin{abstract}
Let $V(n)$ denote the number of positive regular integers $(\bmod n)$ less than or equal to $n$. We give extremal orders of $\frac{V(n) \sigma(n)}{n^{2}}, \frac{V(n) \psi(n)}{n^{2}}$, $\frac{\sigma(n)}{V(n)}, \frac{\psi(n)}{V(n)}$, where $\sigma(n), \psi(n)$ are the sum-of-divisors function and the Dedekind function, respectively. We also give extremal orders for $\frac{\sigma^{*}(n)}{V(n)}$ and $\frac{\phi^{*}(n)}{V(n)}$, where $\sigma^{*}(n)$ and $\phi^{*}(n)$ represent the sum of the unitary divisors of $n$ and the unitary function corresponding to $\phi(n)$, the Euler's function. Finally, we study some extremal orders of compositions $f(g(n))$, involving the functions from above.
\end{abstract}

\section{Introduction}

Let $n>1$ be a positive integer. An integer $a$ is called regular $(\bmod n)$ if there exists an integer $x$ such that $a^{2} x \equiv a(\bmod n)$.

Properties of regular integers have been investigated by several authors. In a recent paper O.Alkam and E.A. Osba [1], using ring theoretic considerations, rediscovered some of the statements proved elementary by J.Morgado [3], [4]. It was proved in [3], [4] that $a>1$ is regular $(\bmod n)$ if and only if $\operatorname{gcd}(a, n)$ is a unitary divisor of $n$. In [11] L.Tóth gives direct proofs of some properties,

Key Words: Arithmetical function, composition, regular integers $(\bmod n)$, extremal orders.

2010 Mathematics Subject Classification: 11A25, 11 N37.

Received:February 2012

Revised:June 2012

Accepted:July 2012 
because the proofs of [3], [4] are lenghty and those of [1] are ring theoretical. Let $\operatorname{Reg}_{n}=\{a: 1 \leq a \leq n$ and a is regular $(\bmod n)\}$, and $V(n)=\# \operatorname{Reg}_{n}$. The function $V$ is multiplicative and $V\left(p^{\alpha}\right)=\phi\left(p^{\alpha}\right)+1=p^{\alpha}-p^{\alpha-1}+1$, where $\phi$ is the Euler function. Consequently, $V(n)=\sum_{d \| n} \phi(d)$, for every $n \geq 1$, where $d \| n$ means unitary divisor (defined later). Also $\phi(n)<V(n) \leq n$, for every $n>1$, and $V(n)=n$ if and only if $n$ is a squarefree, see [4], [11], [1]. L.Tóth [11] proved results concerning the minimal and maximal orders of the functions $V(n)$ and $V(n) / \phi(n)$. The minimal order of $V(n)$ was investigated by O.Alkam and E.A.Osba in [1]. J. Sándor and L. Tóth [7] studied the extremal orders of compositions of certain functions. In the present paper we investigate the extremal orders of the function $V(n)$ in connection with the functions $\sigma(n), \psi(n), \sigma^{*}(n), \phi^{*}(n)$. We also study extremal orders of certain composite functions involving $V(n), \phi(n), \sigma(n), \psi(n), \phi^{*}(n), \sigma^{*}(n)$ and pose some open problems.

For other arithmetic functions defined by regular integers modulo $n$ we refer to the papers [2] and [10].

In what follows let $n=p_{1}^{\alpha_{1}} \cdots p_{k}^{\alpha_{k}}>1$ be a positive integer. We will use throughout the paper the following notation:

- $\quad p_{1}, p_{2}, \ldots$ - the sequence of the primes;

- $\quad d \| n-d$ is a unitary divisor of $n$, that is $d \mid n$ and $\left(d, \frac{n}{d}\right)=1$;

- $\sigma(n)$ - the sum of the divisors of the natural number $n$;

- $\quad \psi(n)$ - the Dedekind function, $\psi(n)=n \prod_{p \mid n}\left(1+\frac{1}{p}\right)$

- $\quad \zeta(n)$ - the Riemann zeta function, $\zeta(s)=\prod_{p \text { prime }}\left(1-\frac{1}{p^{s}}\right)^{-1}, s=\sigma+i t \in$

$\mathbb{C}$ and $\sigma>1$;

- $\quad \phi(n)$ - the Euler function, $\phi(n)=n \prod_{p \mid n}\left(1-\frac{1}{p}\right)$

- $\quad \gamma$ - the Euler constant, $\gamma=\lim _{n \rightarrow \infty}\left(1+\frac{1}{2}+\ldots+\frac{1}{n}-\log n\right)$;

- $\phi^{*}(n)$ - the unitary function corresponding to $\phi(n), \phi^{*}(n)=\prod_{i=1}^{k}\left(p_{i}^{\alpha_{i}}-1\right)$;

- $\sigma^{*}(n)$ - the unitary function corresponding to $\sigma(n), \sigma^{*}(n)=\prod_{i=1}^{k}\left(p_{i}^{\alpha_{i}}+1\right)$. 


\section{Extremal orders concerning classical arithmetic func- tions}

We know that $\phi(n)<n<\sigma(n)$ for every $n>1$. It is easy to see that $\frac{6}{\pi^{2}}<\frac{\phi(n) \sigma(n)}{n^{2}}<1, n>1, \liminf _{n \rightarrow \infty} \frac{\phi(n) \sigma(n)}{n^{2}}=\frac{6}{\pi^{2}}$ and $\limsup _{n \rightarrow \infty} \frac{\phi(n) \sigma(n)}{n^{2}}=1$. In [5] it was proved that $\liminf _{n \rightarrow \infty} \frac{\phi(n) \psi(n)}{n^{2}}=\frac{6}{\pi^{2}}$ and $\limsup _{n \rightarrow \infty} \frac{\phi(n) \psi(n)}{n^{2}}=1$.

We recall that an integer $n>1$ is called powerful if it is divisible by the square of each of its prime factors. A powerful integer is also called a squarefull integer.

The investigation of the minimal and maximal order of $V(n) \sigma(n)$ led us to

\section{Proposition 1.}

$$
\frac{V(n) \sigma(n)}{n^{2}}>1
$$

for every $n>1$.

$$
\begin{gathered}
\liminf _{n \rightarrow \infty} \frac{V(n) \sigma(n)}{n^{2}}=1, \\
\frac{V(n) \sigma(n)}{n^{2}} \leq \frac{\zeta(2)}{\zeta(6)},
\end{gathered}
$$

for every powerful number $n$.

$$
\limsup _{\substack{n \rightarrow \infty \\ n \text { powerful }}} \frac{V(n) \sigma(n)}{n^{2}}=\frac{\zeta(2)}{\zeta(6)}
$$

\section{Proof.}

(i) Let $n>1$ be an integer with the prime factorization $n=p_{1}^{\alpha_{1}} \cdots p_{k}^{\alpha_{k}}$.

Since $\left(1-\frac{1}{p}+\frac{1}{p^{\alpha}}\right) \cdot \frac{p-\frac{1}{p^{\alpha}}}{p-1}>1$, it follows that

$$
\frac{V(n) \sigma(n)}{n^{2}}=\prod_{i=1}^{k}\left(1-\frac{1}{p_{i}}+\frac{1}{p_{i}^{\alpha_{i}}}\right) \cdot \frac{p_{i}-\frac{1}{p_{i}^{\alpha_{i}}}}{p_{i}-1}>1 .
$$

(ii) Since $\lim _{\substack{p \rightarrow \infty \\ p \text { prime }}} \frac{V(p) \sigma(p)}{p^{2}}=\lim _{\substack{p \rightarrow \infty \\ p \text { prime }}} \frac{p^{2}+p}{p^{2}}=1$, taking $(i)$ into account, we obtain

$$
\liminf _{n \rightarrow \infty} \frac{V(n) \sigma(n)}{n^{2}}=1
$$


(iii) Let $n=q_{1}^{\alpha_{1}} \cdots q_{k}^{\alpha_{k}}, q_{1}<q_{2}<\ldots<q_{k}, \alpha_{i} \geq 2,1 \leq i \leq k$ and $p_{1}, \ldots, p_{k}$ the first $k$ primes. We have $\frac{q^{\alpha}-q^{\alpha-1}+1}{q^{\alpha}} \cdot \frac{q^{\alpha+1}-1}{q^{\alpha}(q-1)} \leq \frac{1}{1-\frac{1}{q^{2}}} \cdot\left(1-\frac{1}{q^{6}}\right)$ for $\alpha \geq 2$ and $q$ prime, so

$$
\frac{V(n) \sigma(n)}{n^{2}}=\prod_{i=1}^{k} \frac{q_{i}^{\alpha_{i}}-q_{i}^{\alpha_{i}-1}+1}{q_{i}^{\alpha_{i}}} \cdot \frac{q_{i}^{\alpha_{i}+1}-1}{q_{i}^{\alpha_{i}}\left(q_{i}-1\right)} \leq \prod_{i=1}^{k} \frac{1}{1-\frac{1}{q_{i}^{2}}} \cdot\left(1-\frac{1}{q_{i}^{6}}\right) .
$$

Since $q_{i} \geq p_{i}$ for $1 \leq i \leq k$, it follows that

$$
\begin{aligned}
& \frac{1}{1-\frac{1}{q_{i}^{2}}} \cdot\left(1-\frac{1}{q_{i}^{6}}\right) \leq \frac{1}{1-\frac{1}{p_{i}^{2}}} \cdot\left(1-\frac{1}{p_{i}^{6}}\right) \text { for } 1 \leq i \leq k, \text { so } \\
& \frac{V(n) \sigma(n)}{n^{2}} \leq \prod_{i=1}^{k} \frac{1}{1-\frac{1}{p_{i}^{2}}} \cdot \prod_{i=1}^{k}\left(1-\frac{1}{p_{i}^{6}}\right) .
\end{aligned}
$$

Taking $k \rightarrow \infty$, we obtain

$$
\frac{V(n) \sigma(n)}{n^{2}} \leq \frac{\zeta(2)}{\zeta(6)}
$$

(iv) Taking $n_{k}=p_{1}^{2} \cdots p_{k}^{2}\left(p_{1}, \ldots, p_{k}\right.$ being the first $k$ primes),

$$
\frac{V\left(n_{k}\right) \sigma\left(n_{k}\right)}{n_{k}^{2}}=\prod_{i=1}^{k} \frac{1}{1-\frac{1}{p_{i}^{2}}} \cdot \prod_{i=1}^{k}\left(1-\frac{1}{p_{i}^{6}}\right)
$$

so

$$
\lim _{k \rightarrow \infty} \frac{V\left(n_{k}\right) \sigma\left(n_{k}\right)}{n_{k}^{2}}=\prod_{p \text { prime }} \frac{1}{1-\frac{1}{p^{2}}} \cdot \prod_{p \text { prime }}\left(1-\frac{1}{p^{6}}\right)=\frac{\zeta(2)}{\zeta(6)} .
$$

In view of $(i i i)$, we obtain

$$
\limsup _{\substack{n \rightarrow \infty \\ n \text { powerful }}} \frac{V(n) \sigma(n)}{n^{2}}=\frac{\zeta(2)}{\zeta(6)} .
$$

Corollary 1. The minimal order of $\frac{V(n) \sigma(n)}{n^{2}}$ is 1 and the maximal order of $\frac{V(n) \sigma(n)}{n^{2}}$ for $n$ powerful is $\frac{\zeta(2)}{\zeta(6)}$.

We now prove an analogous result for $V(n) \psi(n)$ : 


\section{Proposition 2.}

$$
\begin{gathered}
\liminf _{\substack{n \rightarrow \infty \\
n \text { squarefree }}} \frac{V(n) \psi(n)}{n^{2}}=1, \\
\frac{V(n) \psi(n)}{n^{2}} \leq \frac{\zeta(3)}{\zeta(6)},
\end{gathered}
$$

for every powerful number $n$.

$$
\limsup _{\substack{n \rightarrow \infty \\ n \text { powerful }}} \frac{V(n) \psi(n)}{n^{2}}=\frac{\zeta(3)}{\zeta(6)} .
$$

\section{Proof.}

(i) Let $n=p_{1} \cdots p_{k}$, where $p_{1}, \ldots, p_{k}$ are distinct prime numbers. We have

$$
\begin{aligned}
& \frac{V(n) \psi(n)}{n^{2}}=\frac{\left(p_{1}+1\right) \cdots\left(p_{k}+1\right)}{p_{1} \cdots p_{k}}>1 \text {. Since } \lim _{\substack{p \rightarrow \infty \\
p \text { prime }}} \frac{V(p) \psi(p)}{p^{2}}=1 \text {, we obtain } \\
& \quad \liminf _{\substack{n \rightarrow \infty \\
n \text { squarefree }}} \frac{V(n) \psi(n)}{n^{2}}=1 .
\end{aligned}
$$

(ii) If $n=q_{1}^{\alpha_{1}} \cdots q_{k}^{\alpha_{k}}, \alpha_{i} \geq 2$, and $1 \leq i \leq k$, then we have

$$
\frac{V(n) \psi(n)}{n^{2}}=\prod_{i=1}^{k} \frac{q_{i}^{\alpha_{i}+1}-q_{i}^{\alpha_{i}-1}+q_{i}+1}{q_{i}^{\alpha_{i}+1}} .
$$

It is immediate that

$\frac{q^{\alpha+1}-q^{\alpha-1}+q+1}{q^{\alpha+1}} \leq\left(1-\frac{1}{q^{2}}\right)\left(1+\frac{1}{q^{2}-q}\right)=1+\frac{1}{q^{3}}$ for $\alpha \geq 2$ and $q$

prime, so

$$
\frac{V(n) \psi(n)}{n^{2}} \leq \prod_{i=1}^{k}\left(1-\frac{1}{q_{i}^{2}}\right)\left(1+\frac{1}{q_{i}^{2}-q_{i}}\right)=\prod_{i=1}^{k}\left(1+\frac{1}{q_{i}^{3}}\right) .
$$

Let $p_{1}, \ldots, p_{k}$ the first $k$ primes. Since $q_{i} \geq p_{i}$ for $1 \leq i \leq k$, we get

$1+\frac{1}{q_{i}^{3}} \leq 1+\frac{1}{p_{i}^{3}}$ for $1 \leq i \leq k$, hence $\frac{V(n) \psi(n)}{n^{2}} \leq \prod_{i=1}^{k}\left(1+\frac{1}{p_{i}^{3}}\right)$. Since the right hand side tends increasingly to $\frac{\zeta(3)}{\zeta(6)}$ as $k \rightarrow \infty$, we get $\frac{V(n) \psi(n)}{n^{2}} \leq \frac{\zeta(3)}{\zeta(6)}$, for every powerful number $n$. 
(iii) Take $n_{k}=p_{1}^{2} \cdots p_{k}^{2}\left(p_{1}, \ldots, p_{k}\right.$ being the first $k$ primes $)$. Then $\frac{V\left(n_{k}\right) \psi\left(n_{k}\right)}{n_{k}^{2}}=\prod_{i=1}^{k}\left(1-\frac{1}{p_{i}^{2}}\right) \cdot \prod_{i=1}^{k}\left(1+\frac{1}{p_{i}^{2}-p_{i}}\right)=\prod_{i=1}^{k}\left(1+\frac{1}{p_{i}^{3}}\right) \rightarrow \frac{\zeta(3)}{\zeta(6)}$,

$(k \rightarrow \infty)$ so, if we take into account $(i i)$, we deduce that

$\limsup _{\substack{n \rightarrow \infty \\ n \text { powerful }}} \frac{V(n) \psi(n)}{n^{2}}=\frac{\zeta(3)}{\zeta(6)}$, implying that the maximal order of $\frac{V(n) \psi(n)}{n^{2}}$

for $n$ powerful is $\frac{\zeta(3)}{\zeta(6)}$.

In order to prove the properties below we apply the following result ([12], Corollary 1) :

Lemma 1. If $f$ is a nonnegative real-valued multiplicative arithmetic function such that for each prime $p$,

(i) $\quad \rho(p)=\sup _{\alpha \geq 0}\left(f\left(p^{\alpha}\right)\right) \leq\left(1-\frac{1}{p}\right)^{-1}$, and

(ii) there is an exponent $e_{p}=p^{o(1)} \in \mathbb{N}$ satisfying $f\left(p^{e_{p}}\right) \geq 1+\frac{1}{p}$,

then $\limsup _{n \rightarrow \infty} \frac{f(n)}{\log \log n}=e^{\gamma} \prod_{p \text { prime }}\left(1-\frac{1}{p}\right) \rho(p)$.

For the quotient $\frac{\sigma(n)}{V(n)}$, we notice that $\frac{\sigma(n)}{V(n)} \geq 1$ for every $n \geq 1$.

Since $\lim _{\substack{p \rightarrow \infty \\ p \text { prime }}} \frac{\sigma(p)}{V(p)}=1$, we get $\liminf _{n \rightarrow \infty} \frac{\sigma(n)}{V(n)}=1$, hence the minimal order of $\frac{\sigma(n)}{V(n)}$ is 1 . Proposition 3 shows that the maximal order of $\frac{\sigma(n)}{V(n)}$ is $e^{2 \gamma}(\log \log n)^{2}$ :

\section{Proposition 3.}

$$
\limsup _{n \rightarrow \infty} \frac{\sigma(n)}{V(n)(\log \log n)^{2}}=e^{2 \gamma}
$$

Proof. Take $f(n)=\sqrt{\frac{\sigma(n)}{V(n)}}$. Then

$$
f\left(p^{\alpha}\right)=\sqrt{\frac{p^{\alpha+1}-1}{(p-1)\left(p^{\alpha}-p^{\alpha-1}+1\right)}} \leq\left(1-\frac{1}{p}\right)^{-1}=\rho(p),
$$


and

$$
f\left(p^{2}\right)=\sqrt{\frac{p^{2}+p+1}{p^{2}-p+1}} \geq 1+\frac{1}{p}
$$

for every prime $p$, so $(i i)$ in the above Lemma is satisfied. We obtain

$$
\limsup _{n \rightarrow \infty} \frac{\sqrt{\sigma(n)}}{\sqrt{V(n)} \log \log n}=e^{\gamma}
$$

so

$$
\limsup _{n \rightarrow \infty} \frac{\sigma(n)}{V(n)(\log \log n)^{2}}=e^{2 \gamma}
$$

Consider now the quotient $\frac{\psi(n)}{V(n)}$. Since $\frac{\psi(n)}{V(n)} \geq 1$ for every $n \geq 1$ and $\frac{\psi(p)}{V(p)}=\frac{p+1}{p}$ for every prime $p$, it is immediate that $\liminf _{n \rightarrow \infty} \frac{\psi(n)}{V(n)}=1$.

Thus, the minimal order of $\frac{\psi(n)}{V(n)}$ is 1 .

\section{Proposition 4.}

$$
\limsup _{n \rightarrow \infty} \frac{\psi(n)}{V(n)(\log \log n)^{2}}=\frac{6}{\pi^{2}} e^{2 \gamma}
$$

Proof. Let $f(n)=\sqrt{\frac{\psi(n)}{V(n)}}$ in Lemma 1. Here

$$
f\left(p^{\alpha}\right)=\sqrt{\frac{p^{\alpha}+p^{\alpha-1}}{p^{\alpha}-p^{\alpha-1}+1}} \leq \sqrt{\frac{p+1}{p-1}}=\rho(p)<\left(1-\frac{1}{p}\right)^{-1}
$$

and

$$
f\left(p^{4}\right)=\sqrt{\frac{p^{4}+p^{3}}{p^{4}-p^{3}+1}} \geq 1+\frac{1}{p},
$$

so $(i i)$ is fulfilled in the cited Lemma, for every prime $p$. We obtain

$$
\limsup _{n \rightarrow \infty} \frac{\sqrt{\psi(n)}}{\sqrt{V(n)} \log \log n}=e^{\gamma} \prod_{p \text { prime }} \sqrt{1-\frac{1}{p^{2}}}=e^{\gamma} \sqrt{\frac{6}{\pi^{2}}}
$$

so

$$
\limsup _{n \rightarrow \infty} \frac{\psi(n)}{V(n)(\log \log n)^{2}}=\frac{6}{\pi^{2}} e^{2 \gamma}
$$




\section{Extremal orders concerning unitary analogues}

In what follows we consider the functions $\sigma^{*}(n)$ and $\phi^{*}(n)$, representing the sum of the unitary divisors of $n$ and the unitary Euler function, respectively. The functions $\sigma^{*}(n)$ and $\phi^{*}(n)$ are multiplicative. If $n=p_{1}^{\alpha_{1}} \cdots p_{k}^{\alpha_{k}}$ is the prime factorisation of $n>1$, then

$$
\phi^{*}(n)=\left(p_{1}^{\alpha_{1}}-1\right) \cdots\left(p_{k}^{\alpha_{k}}-1\right), \quad \sigma^{*}(n)=\left(p_{1}^{\alpha_{1}}+1\right) \cdots\left(p_{k}^{\alpha_{k}}+1\right)
$$

Note that $\sigma^{*}(n)=\sigma(n), \phi^{*}(n)=\phi(n)$ for all squarefree $n$, and for every $n \geq 1$

$$
\phi(n) \leq \phi^{*}(n) \leq n \leq \sigma^{*}(n) \leq \sigma(n)
$$

We give extremal orders for the quotients $\frac{\sigma^{*}(n)}{V(n)}$ and $\frac{\phi^{*}(n)}{V(n)}$, the minimal order of $\frac{\phi^{*}(n)}{V(n)}$ being studied for powerful numbers. Since $\frac{\sigma^{*}(n)}{V(n)} \geq 1$ and for prime numbers $p, \lim _{p \rightarrow \infty} \frac{\sigma^{*}(p)}{V(p)}=\lim _{p \rightarrow \infty} \frac{p+1}{p}=1$, it follows that $\liminf _{n \rightarrow \infty} \frac{\sigma^{*}(n)}{V(n)}=1$.

If $n$ is powerful, it is easy to see that $\frac{\phi^{*}(n)}{V(n)} \geq 1$, taking into account that $\frac{\phi^{*}\left(p^{\alpha}\right)}{V\left(p^{\alpha}\right)} \geq 1$ for $\alpha \geq 2$. For prime numbers $p$, we notice that $\lim _{p \rightarrow \infty} \frac{\phi^{*}\left(p^{2}\right)}{V\left(p^{2}\right)}=$ $\lim _{p \rightarrow \infty} \frac{p^{2}-1}{p^{2}-p+1}=1$, which implies that $\liminf _{n \rightarrow \infty} \frac{\phi^{*}(n)}{V(n)}=1$, so the minimal order of $\frac{\phi^{*}(n)}{V(n)}$ is 1 . For the maximal orders of these quotients we give:

\section{Proposition 5.}

$$
\begin{aligned}
& \limsup _{n \rightarrow \infty} \frac{\sigma^{*}(n)}{V(n) \log \log n}=e^{\gamma}, \\
& \limsup _{n \rightarrow \infty} \frac{\phi^{*}(n)}{V(n) \log \log n}=e^{\gamma} .
\end{aligned}
$$

Proof.

(i) Take $f(n)=\frac{\sigma^{*}(n)}{V(n)}$, which is a nonnegative real-valued multiplicative arithmetic function. We have $f\left(p^{\alpha}\right)=\frac{p^{\alpha}+1}{p^{\alpha}-p^{\alpha-1}+1} \leq\left(1-\frac{1}{p}\right)^{-1}=\rho(p)$, and 
$f(p)=1+\frac{1}{p} \geq 1+\frac{1}{p}$ for every prime $p$. Applying Lemma 1 , we get

$$
\limsup _{n \rightarrow \infty} \frac{\sigma^{*}(n)}{V(n) \log \log n}=e^{\gamma}
$$

(ii) Now let $f(n)=\frac{\phi^{*}(n)}{V(n)}$. Here

$f\left(p^{\alpha}\right)=\frac{p^{\alpha}-1}{p^{\alpha}-p^{\alpha-1}+1} \leq\left(1-\frac{1}{p}\right)^{-1}=\rho(p)$, and

$f\left(p^{4}\right)=\frac{p^{4}-1}{p^{4}-p^{3}+1} \geq 1+\frac{1}{p}$, for every prime $p$. According to Lemma 1 ,

$$
\limsup _{n \rightarrow \infty} \frac{\phi^{*}(n)}{V(n) \log \log n}=e^{\gamma}
$$

Corollary 2. The maximal order of both $\frac{\sigma^{*}(n)}{V(n)}$ and $\frac{\phi^{*}(n)}{V(n)}$ is $e^{\gamma} \log \log n$.

\section{Extremal orders regarding compositions of functions}

We now move to the study of extremal orders of some composite arithmetic functions. We start with $V(V(n))$ and $\phi(V(n))$.

We know that $V(n) \leq n$ for every $n \geq 1$, so $\frac{V(V(n))}{n} \leq \frac{V(n)}{n} \leq 1$ and $\lim _{\substack{p \rightarrow \infty \\ p \text { prime }}} \frac{V(V(p))}{p}=\lim _{\substack{p \rightarrow \infty \\ p \text { prime }}} \frac{V(p)}{p}=1$, so the maximal order of $V(V(n))$ is $n$. Since $\phi(n) \leq n$ and $V(n) \leq n$ for any $n \geq 1$, we have $\frac{\phi(V(n))}{n} \leq \frac{V(n)}{n} \leq 1$. But $\lim _{\substack{p \rightarrow \infty \\ p \text { prime }}} \frac{\phi(V(p))}{p}=\lim _{p \rightarrow \infty} \frac{p-1}{p}=1$, so the maximal order of $\phi(V(n))$ is $n$. In [7] was investigated the maximal order of $\phi^{*}(\phi(n))$. Using the general idea of that proof, we show:

Proposition 6. The maximal order of $V(\phi(n))$ is $n$.

Proof. We will use Linnik's theorem which states that if $(k, \ell)=1$, then there exists a prime $p$ such that $p \equiv \ell(\bmod k)$ and $p \ll k^{c}$, where $c$ is a constant (one can take $c \leq 11$ ).

Let $A=\prod_{\substack{p \leq x \\ p \text { prime }}} p$. Since $\left(A^{2}, A+1\right)=1$, by Linnik's theorem there is a prime number $q$ such that $q \equiv A+1\left(\bmod A^{2}\right)$ and $q \ll\left(A^{2}\right)^{c}=A^{2 c}$, where $c$ 
satisfies $c \leq 11$. Let $q$ be the least prime satisfying the above condition. So, $q-A-1=k A^{2}$, for some $k$. We have

$\phi(q)=q-1=A+k A^{2}=A(1+k A)=A B$, where $B=1+k A$. Thus

$(A, B)=1$, so $\mathrm{B}$ is free of prime factors $\leq x$. We have $q-1=A B$, so $q=A B+1$.

Since $V(n)$ is multiplicative, we have

$$
\frac{V(\phi(q))}{q}=\frac{V(A B)}{A B+1}=\frac{V(A)}{A} \cdot \frac{V(B)}{B} \cdot \frac{A B}{A B+1} .
$$

Here $\frac{A B}{A B+1} \rightarrow 1$ as $x \rightarrow \infty$, so it is sufficient to study $\frac{V(A)}{A}$ and $\frac{V(B)}{B}$. Clearly,

$$
\frac{V(A)}{A}=\frac{V\left(\prod_{p \leq x} p\right)}{\prod_{p \leq x} p}=\frac{\prod_{p \leq x} V(p)}{\prod_{p \leq x} p}=1 .
$$

It is well-known that $A=\prod_{p \leq x} p=e^{O(x)}$. Since $q \ll A^{2 c}$ and $A=e^{O(x)}$, from $B \ll A^{10}$ we have $B \ll\left(e^{O(x)}\right)^{10}=e^{O(x)}$, so

$$
\log B \ll x
$$

If $B=\prod_{i=1}^{k} q_{i}^{b_{i}}$ is the prime factorization of $B$, we obtain $\log B=\sum_{i=1}^{k} b_{i} \log q_{i}>(\log x) \sum_{i=1}^{k} b_{i}$, as $q_{i}>x$ for all $i \in\{1,2, \ldots, k\}$. But $\sum_{i=1}^{k} b_{i} \geq k$, so $\log B>k \log x$, implying that $k<\frac{\log B}{\log x} \ll \frac{x}{\log x}($ by $(3))$. We get:

$$
\begin{aligned}
& \frac{V(B)}{B}=\frac{V\left(\prod_{i=1}^{k} q_{i}^{b_{i}}\right)}{\prod_{i=1}^{k} q_{i}^{b_{i}}}=\frac{\prod_{i=1}^{k}\left(q_{i}^{b_{i}}-q_{i}^{b_{i}-1}+1\right)}{\prod_{i=1}^{k} q_{i}^{b_{i}}}>\frac{\prod_{i=1}^{k}\left(q_{i}^{b_{i}}-q_{i}^{b_{i}-1}\right)}{\prod_{i=1}^{k} q_{i}^{b_{i}}}= \\
& =\prod_{i=1}^{k}\left(1-\frac{1}{q_{i}}\right)>\left(1-\frac{1}{x}\right)^{k} \geq\left(1-\frac{1}{x}\right)^{O\left(\frac{x}{\log x}\right)}>1+O\left(\frac{1}{\log x}\right)
\end{aligned}
$$


because $1-\frac{1}{q_{i}}>1-\frac{1}{x}$. So,

$$
\frac{V(B)}{B}>1+O\left(\frac{1}{\log x}\right)
$$

By (1), (2), (4) and $\frac{A B}{A B+1} \rightarrow 1$ as $x \rightarrow \infty$, we obtain

$$
\frac{V(\phi(q))}{q}>1+O\left(\frac{1}{\log x}\right) .
$$

By relation (5), and since $\frac{V(\phi(n))}{n} \leq \frac{\phi(n)}{n} \leq 1$, it follows that

$$
\limsup _{n \rightarrow \infty} \frac{V(\phi(n))}{n}=1 .
$$

Proposition 7. The maximal order of $V\left(\phi^{*}(n)\right)$ is $n$.

Proof. We apply the following result:

If $a$ is an integer, $a>1, p$ is a prime number and $f(n)$ is an arithmetical function satisfying $\phi(n) \leq f(n) \leq \sigma(n)$, one has

$$
\lim _{p \rightarrow \infty} \frac{f(N(a, p))}{N(a, p)}=1,
$$

where $N(a, p)=\frac{a^{p}-1}{a-1}$ (see e.g. D.Suryanarayana [9]).

Since $\phi^{*}(n) \leq n$, it follows that $V\left(\phi^{*}(n)\right) \leq \phi^{*}(n) \leq n$, so

$$
\frac{V\left(\phi^{*}(n)\right)}{n} \leq 1
$$

Let $n=2^{p}, p$ prime number. Then we have

$$
\frac{V\left(\phi^{*}\left(2^{p}\right)\right)}{2^{p}}=\frac{V\left(2^{p}-1\right)}{2^{p}-1} \cdot \frac{2^{p}-1}{2^{p}} .
$$

Since $\phi(n) \leq V(n) \leq \sigma(n)$ and $N(2, p)=2^{p}-1$, it follows that

$$
\lim _{p \rightarrow \infty} \frac{V\left(2^{p}-1\right)}{2^{p}-1}=1,
$$

taking into account (6). By (8), taking $p \rightarrow \infty$, we obtain

$$
\lim _{p \rightarrow \infty} \frac{V\left(\phi^{*}\left(2^{p}\right)\right)}{2^{p}}=1 .
$$


Now (7) and (9) imply $\limsup _{n \rightarrow \infty} \frac{V\left(\phi^{*}(n)\right)}{n}=1$.

For the maximal orders of $\frac{\sigma\left(\phi^{*}(n)\right)}{V\left(\phi^{*}(n)\right)}, \frac{\psi\left(\phi^{*}(n)\right)}{V\left(\phi^{*}(n)\right)}$ we give

\section{Proposition 8.}

(i) $\limsup _{n \rightarrow \infty} \frac{\sigma\left(\phi^{*}(n)\right)}{V\left(\phi^{*}(n)\right)(\log \log n)^{2}}=\limsup _{n \rightarrow \infty} \frac{\sigma\left(\phi^{*}(n)\right)}{V\left(\phi^{*}(n)\right)\left(\log \log \phi^{*}(n)\right)^{2}}=e^{2 \gamma}$,

(ii) $\limsup _{n \rightarrow \infty} \frac{\psi\left(\phi^{*}(n)\right)}{V\left(\phi^{*}(n)\right)(\log \log n)^{2}}=\limsup _{n \rightarrow \infty} \frac{\psi\left(\phi^{*}(n)\right)}{V\left(\phi^{*}(n)\right)\left(\log \log \phi^{*}(n)\right)^{2}}=\frac{6}{\pi^{2}} e^{\gamma}$.

\section{Proof.}

(i) Let

$$
l_{1}:=\limsup _{n \rightarrow \infty} \frac{\sigma\left(\phi^{*}(n)\right)}{V\left(\phi^{*}(n)\right)(\log \log n)^{2}}
$$

and

$$
l_{2}:=\limsup _{n \rightarrow \infty} \frac{\sigma\left(\phi^{*}(n)\right)}{V\left(\phi^{*}(n)\right)\left(\log \log \phi^{*}(n)\right)^{2}} .
$$

Since $\phi^{*}(n) \leq n$ for every $n \geq 1$,

$l_{1}=\limsup _{n \rightarrow \infty} \frac{\sigma\left(\phi^{*}(n)\right)}{V\left(\phi^{*}(n)\right)(\log \log n)^{2}} \leq l_{2}=\limsup _{n \rightarrow \infty} \frac{\sigma\left(\phi^{*}(n)\right)}{V\left(\phi^{*}(n)\right)\left(\log \log \phi^{*}(n)\right)^{2}} \leq$

$\limsup _{m \rightarrow \infty} \frac{\sigma(m)}{V(m)(\log \log m)^{2}}=e^{2 \gamma}$, by Proposition 3 .

Since $(n, 1)=1$, by Linnik's theorem, there exists a prime number $p$ such that $p \equiv 1(\bmod n)$ and $p \ll n^{c}$. Let $p_{n}$ be the least prime such that $p_{n} \equiv 1$ $(\bmod n)$, for every $n$. Then $n \mid p_{n}-1$ and $p_{n} \ll n^{c}$, so $\log \log p_{n} \sim \log \log n$. Observe that $a \mid b$ implies $\frac{\sigma(a)}{V(a)} \leq \frac{\sigma(b)}{V(b)}$. If $p^{\beta} \mid p^{\alpha}(\beta \leq \alpha)$, it is easy to see that $\frac{\sigma\left(p^{\beta}\right)}{V\left(p^{\beta}\right)} \leq \frac{\sigma\left(p^{\alpha}\right)}{V\left(p^{\alpha}\right)}$. The general case follows, taking into account that $\frac{\sigma(n)}{V(n)}$ is multiplicative. So,

$$
\begin{aligned}
& \frac{\sigma\left(\phi^{*}\left(p_{n}\right)\right)}{V\left(\phi^{*}\left(p_{n}\right)\right)\left(\log \log p_{n}\right)^{2}}= \\
& \frac{\sigma\left(p_{n}-1\right)}{V\left(p_{n}-1\right)\left(\log \log p_{n}\right)^{2}} \sim \frac{\sigma\left(p_{n}-1\right)}{V\left(p_{n}-1\right)(\log \log n)^{2}} \geq \frac{\sigma(n)}{V(n)(\log \log n)^{2}} .
\end{aligned}
$$

But

$\limsup _{n \rightarrow \infty} \frac{\sigma\left(\phi^{*}(n)\right)}{V\left(\phi^{*}(n)\right)(\log \log n)^{2}} \geq \limsup _{n \rightarrow \infty} \frac{\sigma\left(\phi^{*}\left(p_{n}\right)\right)}{V\left(\phi^{*}\left(p_{n}\right)\right)\left(\log \log p_{n}\right)^{2}} \geq$ 
$\limsup _{n \rightarrow \infty} \frac{\sigma(n)}{V(n)(\log \log n)^{2}}=e^{2 \gamma}$

We obtain $e^{2 \gamma} \leq l_{1} \leq l_{2} \leq e^{2 \gamma}$, hence $l_{1}=l_{2}=e^{2 \gamma}$.

(ii) The proof is similar to the proof of $(i)$, taking into account that $a \mid b$ impies $\frac{\psi(a)}{V(a)} \leq \frac{\psi(b)}{V(b)}$ and $\limsup _{n \rightarrow \infty} \frac{\psi(n)}{V(n)(\log \log n)^{2}}=\frac{6}{\pi^{2}} e^{2 \gamma}$, by Proposition 4 .

So, the maximal orders of $\frac{\sigma\left(\phi^{*}(n)\right)}{V\left(\phi^{*}(n)\right)}, \frac{\psi\left(\phi^{*}(n)\right)}{V\left(\phi^{*}(n)\right)}$ are $e^{2 \gamma}(\log \log n)^{2}$ and $\frac{6}{\pi^{2}} e^{2 \gamma}(\log \log n)^{2}$, respectively. In a similar manner, since $\limsup _{n \rightarrow \infty} \frac{\sigma^{*}(n)}{V(n) \log \log n}=\limsup _{n \rightarrow \infty} \frac{\phi^{*}(n)}{V(n) \log \log n}=e^{\gamma}$ (Proposition 5), $a \mid b$ implies $\frac{\sigma^{*}(a)}{V(a)} \leq \frac{\sigma^{*}(b)}{V(b)}$ and $\frac{\phi^{*}(a)}{V(a)} \leq \frac{\phi^{*}(b)}{V(b)}$, respectively, it can be shown that $\limsup _{n \rightarrow \infty} \frac{\sigma^{*}\left(\phi^{*}(n)\right)}{V\left(\phi^{*}(n)\right) \log \log n}=\limsup _{n \rightarrow \infty} \frac{\sigma^{*}\left(\phi^{*}(n)\right)}{V\left(\phi^{*}(n)\right) \log \log \phi^{*}(n)}=e^{\gamma}$ and $\limsup _{n \rightarrow \infty} \frac{\phi^{*}\left(\phi^{*}(n)\right)}{V\left(\phi^{*}(n)\right) \log \log n}=\limsup _{n \rightarrow \infty} \frac{\phi^{*}\left(\phi^{*}(n)\right)}{V\left(\phi^{*}(n)\right) \log \log \phi^{*}(n)}=e^{\gamma}$.

\section{Open Problems}

Problem 1. Note that

$$
\limsup _{n \rightarrow \infty} \frac{V(n) \sigma(n)}{n^{2}}=\limsup _{n \rightarrow \infty} \frac{V(n) \psi(n)}{n^{2}}=\infty,
$$

since for $n_{k}=p_{1} \cdots p_{k}$ (the product of the first $k$ primes),

$$
\frac{V\left(n_{k}\right) \sigma\left(n_{k}\right)}{n_{k}^{2}}=\frac{\left(p_{1}+1\right) \cdots\left(p_{k}+1\right)}{p_{1} \cdots p_{k}}=\prod_{i=1}^{k}\left(1+\frac{1}{p_{i}}\right) \rightarrow \infty, k \rightarrow \infty ;
$$

the other relation follows in a similar manner. What are the maximal orders for $\frac{V(n) \sigma(n)}{n^{2}}$ and $\frac{V(n) \psi(n)}{n^{2}}$ ?

Problem 2. Note that

$$
\liminf _{n \rightarrow \infty} \frac{V(\phi(n))}{n}=\liminf _{n \rightarrow \infty} \frac{V\left(\phi^{*}(n)\right)}{n}=\liminf _{n \rightarrow \infty} \frac{\phi^{*}(V(n))}{n}=0 .
$$


For $n_{k}=p_{1} \cdots p_{k}$ (the product of the first $k$ primes),

$$
\begin{gathered}
\frac{V\left(\phi\left(n_{k}\right)\right)}{n_{k}}=\frac{V\left(\left(p_{1}-1\right) \cdots\left(p_{k}-1\right)\right)}{p_{1} \cdots p_{k}} \leq \frac{\left(p_{1}-1\right) \cdots\left(p_{k}-1\right)}{p_{1} \cdots p_{k}} \\
=\left(1-\frac{1}{p_{1}}\right) \cdots\left(1-\frac{1}{p_{k}}\right)
\end{gathered}
$$

so

$$
\lim _{k \rightarrow \infty} \frac{V\left(\phi\left(n_{k}\right)\right)}{n_{k}}=\lim _{k \rightarrow \infty}\left(1-\frac{1}{p_{1}}\right) \cdots\left(1-\frac{1}{p_{k}}\right)=0,
$$

similarly the other relations. What are the minimal orders for the $V(\phi(n))$, $V\left(\phi^{*}(n)\right), \phi^{*}(V(n))$ ?

Problem 3. Taking $n_{k}=p_{1} \cdots p_{k}$ (the product of the first $k$ primes),

$$
\begin{gathered}
\frac{\sigma^{*}\left(V\left(n_{k}\right)\right)}{n_{k}}=\frac{\sigma^{*}\left(p_{1} \cdots p_{k}\right)}{p_{1} \cdots p_{k}}=\frac{\left(p_{1}+1\right) \cdots\left(p_{k}+1\right)}{p_{1} \cdots p_{k}} \\
=\left(1+\frac{1}{p_{1}}\right) \cdots\left(1+\frac{1}{p_{k}}\right) \rightarrow \infty
\end{gathered}
$$

as $k \rightarrow \infty$, so $\limsup _{n \rightarrow \infty} \frac{\sigma^{*}(V(n))}{n}=\infty$. What is the maximal order for $\sigma^{*}(V(n)) ?$

ACKNOWLEDGEMENT. The author would like to thank Professor L.Tóth and Professor G. Mincu for the valuable suggestions which improved the presentation.

The author also thanks the referees for helpful suggestions.

\section{References}

[1] O. Alkam, E. Osba, On the regular elements in $\mathbb{Z}_{n}$, Turk.J.Math., $32(2008)$.

[2] P. Haukkanen, L. Tóth, An analogue of Ramanujan's sum with respect to regular integers $(\bmod r)$, Ramanujan J., 27(2012) 71-88.

[3] J. Morgado, A property of the Euler $\phi$-function concerning the integers which are regular modulo n, Portugal. Math., 33 (1974), 185-191. 
[4] J. Morgado, Inteiros regulares módulo n, Gazeta de Matematica (Lisboa), $33(1972)$, No. 125-128, 1-5.

[5] J. Sándor, Geometric Theorems, Diophantine Equations, And Arithmetic Functions, American Research Press Rehoboth, 2002.

[6] J. Sándor, B. Crstici, Handbook of number theory II, Kluwer Academic Publishers, Dordrecht, 2004.

[7] J. Sándor, L. Tóth, Extremal orders of compositions of certain arithmetical functions, Integers: Electronic Journal of Combinatorial Number Theory, 8 (2008), \# A34.

[8] R. Sivaramakrishnan, Classical theory of arithmetic functions, Monographs and Textbooks in Pure and Applied Mathematics, 126 . Marcel Dekker, Inc., New York, 1989.

[9] D. Suryanarayana, On a class of sequences of integers, Amer. Math. Monthly, 84(1977), 728-730.

[10] L. Tóth, A gcd-sum function over regular integers modulo $n$, J. Integer Seq., 12(2009), no.2, Article 09.2.5, 8 pp.

[11] L. Tóth, Regular integers $(\bmod n)$, Annales Univ. Sci. Budapest., Sect Comp., 29(2008), 264-275, see http://front.math.ucdavis.edu/0710.1936.

[12] L. Tóth, E. Wirsing, The maximal order of a class of multiplicative arithmetical functions, Annales Univ. Sci. Budapest., Sect Comp., $22(2003)$, 353-364.

Brăduţ APOSTOL,

"Spiru Haret" Pedagogical High School,

1 Timotei Cipariu St. , RO - 620004 Focşani, ROMANIA,

Email:apo_brad@yahoo.com 